\title{
Benefits of stereotactic ablative radiotherapy combined with incomplete transcatheter arterial chemoembolization in hepatocellular carcinoma
}

Eun Kyung Paik', Mi-Sook Kim", Won II Jang ${ }^{1}$, Young Seok Seo ${ }^{1}$, Chul-Koo Cho ${ }^{1}$, Hyung Jun Yoo ${ }^{1}$, Chul Ju Han², Su Cheol Park², Sang Bum Kim ${ }^{3}$ and Young Han Kim ${ }^{4}$

\begin{abstract}
Background: This study aimed to evaluate the effect of stereotactic ablative radiotherapy (SABR) after incomplete transcatheter arterial chemoembolization (TACE) in hepatocellular carcinoma (HCC) patients.

Methods: The study enrolled 178 HCC patients initially treated with TACE between 2006 and 2011. Patients were included if they had Barcelona Clinic Liver Cancer stage 0 or $A, \leq 3$ nodules with a total sum of longest diameter $\leq 10 \mathrm{~cm}$, Child-Turcotte-Pugh score of $\leq 7$, no major vessel invasion, and no extra-hepatic metastases.

Results: Twenty-four patients achieved a complete response to TACE (group 1). Among those with incomplete response, 47 patients received other curative treatments (group 2), 37 received SABR (group 3), and 70 received non-curative treatments (group 4). The 2-year overall survival (OS) rates for groups 1, 2, 3, and 4 were $88 \%, 81 \%$, $73 \%$, and $54 \%$, respectively. The corresponding 5 -year OS rates were $50 \%, 58 \%, 53 \%$, and $28 \%$, respectively.

Conclusions: Patients treated with SABR after incomplete TACE had similar survival outcomes to those achieving complete response to TACE or receiving curative treatments. However, patients receiving non-curative treatments had significantly lower survival rates than the other groups. Therefore, if SABR was indicated at the initial diagnosis, it might be recommended after TACE failure.
\end{abstract}

Keywords: Hepatocellular carcinoma, Radiotherapy, Stereotactic ablative radiotherapy, Transcatheter arterial chemoembolization

\section{Background}

The primary treatment for hepatocellular carcinoma (HCC) is surgery, including hepatic resection and liver transplantation, which results in 5-year survival rates of $30-70 \%$ [1]. However, $<20 \%$ of HCC patients are eligible for surgery. In unresectable cases, local therapies such as radiofrequency ablation (RFA) or percutaneous ethanol injection (PEI) are reportedly effective, but not all patients are eligible for these treatments due to the tumor's percutaneous inaccessibility, its invisibility via

\footnotetext{
* Correspondence: mskim@kirams.re.kr

${ }^{1}$ Department of Radiation Oncology, Korea Cancer Center Hospital, Korea Institute of Radiological and Medical Sciences, 75 Nowon-ro, Nowon-gu, Seoul, Republic of Korea

Full list of author information is available at the end of the article
}

ultrasonography, or bleeding risks. Transcatheter arterial chemoembolization (TACE) has been widely used as the first-line non-curative therapy for HCC cases that are non-surgical or unsuitable for local ablative therapies [2]. However, TACE alone rarely produces a complete response, and additional treatments are often required. Multiple repetitive sessions of TACE have been widely performed in Korea, but such treatments deteriorate liver function, increase TACE-related adverse effects, and offer less therapeutic efficacy due to vascularity decrease [3]. Therefore, various modalities such as RFA, PEI, sorafenib, conventional radiotherapy (RT), and stereotactic ablative radiotherapy (SABR) have been suggested in addition to TACE, but a definitive guideline has not been established $[4,5]$. 
The role of RT in HCC is limited owing to the liver's low tolerance to radiation and the risk of radiationinduced liver damage $[6,7]$. However, recent radiotherapeutic developments have gradually expanded the indications for external beam radiotherapy from palliative to curative with high doses of radiation safely delivered to the tumor while avoiding adverse effects to the liver function. In several studies on three-dimensional conformal radiotherapy (3D-CRT) to primary $\mathrm{HCC}$, substantial effects of RT have been observed [8-10]. With the introduction of SABR, it is now possible to accurately deliver more radiation doses to tumors using fewer fractions while sparing the normal liver tissue [11, 12]. Recent clinical data have demonstrated the feasibility of SABR for HCC treatment with high local control (LC) and overall survival (OS) rates and low treatment-related severe toxicities [13-21].

However, there remains a lack of randomized studies comparing the effects of TACE combined with RT and those of TACE alone. Although several phase II trials of SABR have reported outstanding results, including LC rates of $>90 \%$ and 5 -year OS of $>50 \%$ in well selected cases of HCC, RT is still not endorsed as a curative treatment option for HCC in most guidelines or consensus strategies [1, 22, 23]. Therefore, this retrospective study aimed to provide the basis for initiating a randomized trial to investigate whether a combination of SABR and TACE would improve the long-term OS compared with TACE alone. The survival outcomes of TACE plus SABR were compared with those of other treatment modalities combined with TACE or TACE alone.

\section{Methods}

\section{Patients}

Between January 2006 and December 2011, a total of 832 consecutive $\mathrm{HCC}$ patients were treated with TACE at Korea Institute of Radiological and Medical Sciences. Of those, patients receiving curative treatments such as surgery or RFA at initial diagnosis were excluded, and only those initially treated with TACE were included in this study. All patients' medical records were retrospectively reviewed. A total of 178 patients met the following eligibility criteria: (1) Barcelona Clinic Liver Cancer (BCLC) stage 0 or $\mathrm{A},(2)$ single or multiple lesions $(\leq 3$ nodules), (3) each tumor measuring $\leq 10 \mathrm{~cm}$ at the longest diameter (LD), with the sum of LD being $\leq 10 \mathrm{~cm}$, (4) Child-Turcotte-Pugh (CTP) score of $\leq 7$, (5) no major vessel invasion, and (6) no extrahepatic metastases. The exclusion criteria included (1) diffuse infiltrative tumor type, (2) liver cirrhosis-associated complications such as uncontrolled ascites or encephalopathy, (3) severe comorbidities, (4) previous RT to the upper abdomen, and (5) other malignancies within 5 years. These are our institutional criteria for curative SABR in HCC patients $[4,19]$. This study was approved by our institutional review board.

\section{TACE}

TACE was performed with an infusion of a lipiodol and doxorubicin mixture. Tumor response to TACE was evaluated using computed tomography (CT) 1 month after TACE. Although the definition of TACE failure has been proposed by the Japan Society of Hepatology [23], there is no internationally accepted consensus on the definition of TACE failure or TACE refractoriness criteria. In our study, incomplete response to TACE was defined as disease progression, incomplete tumor filling by the lipiodol-doxorubicin mixture on CT images. Disease progression included residual viable tumors, progression of existing lesions, or development of new lesions. Patients' final response to TACE was assessed by a hepatologist. Although current evidence suggests that 1 cycle of TACE may not be sufficient for effective treatment and repeating TACE prolongs survival [24], no guidelines are available on the criteria for repeating TACE. In this study, if TACE yielded a complete response, no additional treatment was given, and the patient was followed up by regular evaluations. In cases of incomplete response, additional TACE was performed, or a change in treatment strategy was conducted.

\section{SABR}

SABR was performed as previously described [4, 19]. Patients were treated using either a CyberKnife (Accuray Inc., Sunnyvale, CA, USA) or a RapidArc (Varian Medical Systems, Palo Alto, CA, USA) system. Gold fiducials inserted near the spine closest to the lesion were used for tracking in patients treated with CyberKnife. For patients treated with RapidArc, gold fiducials inserted in the liver near the tumor or lipiodol uptake were used as markers for image guidance with the cone-beam CT. To compensate for breathing motion, simulation CT with abdominal compression was performed, and both slow and helical CT images were obtained with contrast medium. The gross tumor volume (GTV) was defined in the slow CT set as the viable tumor with the contrast medium uptake including the embolization material. These slow CT images included the respiratory movement of the target, therefore the GTV used for planning was larger than the actual gross tumor and was referred to as the internal target volume (ITV). To better delineate the tumor volumes, magnetic resonance imaging (MRI) images were used as references on a regular basis. A margin of 0-4 mm was added to the ITV for the planning target volume (PTV). SABR doses were prescribed at an isodose line (70-80\% of the maximum dose) that covered at least $97 \%$ of the PTV. 
Treatment doses were determined according to previously described protocols $[4,19,20]$. A total dose of $40-$ 60 Gy (median 56 Gy) in 3-5 fractions was prescribed. Briefly summarizing the previous protocols, the final prescribed doses were 60 Gy in 3 fractions, but the dosages were reduced by 0.5 or 1 Gy per fraction until the normal tissue constraints were allowed. For the normal liver, at least $700 \mathrm{ml}$ of normal liver (entire liver minus the cumulative GTV) should not receive a total dose $\geq 17$ Gy in 3 fractions. For the spinal cord, the maximum dose should not exceed 18 Gy in 3 fractions. For the esophagus, the maximum dose should not exceed 24 Gy in 3 fractions. In addition, although other normal tissue constraints were not considered, dosages to the kidney, intestine, and stomach were restricted to the lowest levels possible.

\section{Evaluation and statistical analysis}

Baseline patient and tumor characteristics were compared among groups using the one-way analysis of variance or the Kruskal-Wallis test as appropriate. Survival was calculated from the date of the initial TACE using the KaplanMeier method, and intergroup comparisons were performed using the log-rank test. Univariate analysis was performed using the log-rank test to identify the significant prognostic factors for survival. The Cox regression model was applied to all potentially significant variables for the multivariate analysis. For all analyses, two-sided tests of significance were used with $P$ values $<0.05$ considered significant. All statistical analyses were performed using the Statistical Package for the Social Sciences (version 14.0; SPSS, Inc. Chicago, IL, USA).

\section{Results}

\section{Baseline patient characteristics}

Of the 832 consecutive HCC patients treated with TACE, 178 met all eligibility criteria. Our enrollment suggests that among patients currently treated with TACE, approximately $21 \%$ (or higher if those initially treated with surgery or other local ablative therapies are included) may be eligible for SABR. Twenty-four (14\%) patients achieved a complete response to TACE as the primary treatment (group 1; complete TACE group). The remaining 154 ( $86 \%$ ) patients showed incomplete response and were further treated with other modalities. Of these, 47 (26\%) patients further received curative treatments (group 2; incomplete TACE + curative Tx group) such as surgery, RFA, or PEI; 37 (21 \%) were treated with SABR (group 3; incomplete TACE + SABR group); and 70 (39\%) received non-curative treatments (group 4; incomplete TACE + non-curative Tx group) such as repeated TACE, sorafenib, and chemotherapy (Fig. 1). Among the 47 group 2 patients, 35 (75\%) received surgery including resection and liver transplantation, $11(23 \%)$ received RFA, and 1 (2\%) received PEI. Among the 70 group 4 patients, 66 (95\%) received repeated TACE, $3(4 \%)$ received sorafenib, and $1(1 \%)$ received chemotherapy. The median number (range) of previous TACE sessions performed for groups $1,2,3$, and 4 patients were 1 (1-4), 1 (1-5), 2 (1-6), and 2 (1-13), respectively.

No significant differences in age, sex, BCLC stage, and tumor size were observed among the 4 groups (Table 1 ). However, statistically significant differences in CTP scores were observed between groups 2 and $4(P=0.004)$. Furthermore, patient characteristics did not differ among

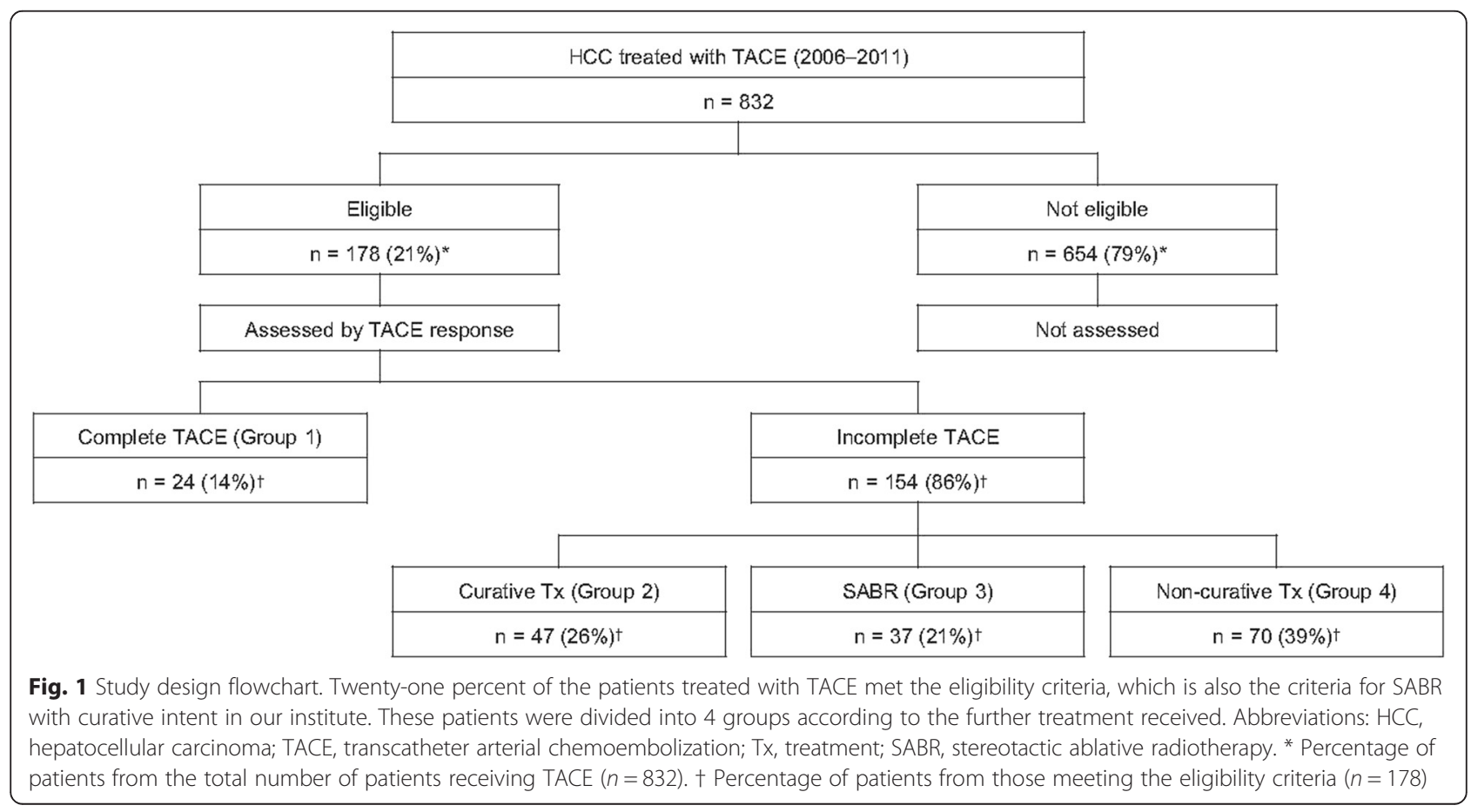


Table 1 Number of patients (\%) for patient and tumor characteristics of each treatment group

\begin{tabular}{|c|c|c|c|c|c|}
\hline \multirow[t]{2}{*}{ Characteristics } & \multirow{2}{*}{$\begin{array}{l}\text { Complete } \\
\text { TACE }\end{array}$} & \multicolumn{3}{|c|}{ Incomplete TACE } & \multirow[t]{2}{*}{$P$-value } \\
\hline & & Curative Tx & SABR & Non-curative Tx & \\
\hline Age & & & & & 0.622 \\
\hline Range (median, years) & $41-83(55)$ & $35-77(60)$ & $42-74(59)$ & 24-84 (59) & \\
\hline Sex & & & & & 0.426 \\
\hline Male & $14(58)$ & $33(70)$ & $29(78)$ & $49(70)$ & \\
\hline Female & $10(42)$ & $14(30)$ & $8(22)$ & $21(30)$ & \\
\hline BCLC stage & & & & & 0.135 \\
\hline 0 & $9(38)$ & $8(17)$ & $6(16)$ & $12(17)$ & \\
\hline A & $15(63)$ & $39(83)$ & $31(84)$ & $58(83)$ & \\
\hline CTP score & & & & & 0.040 \\
\hline 5 & $18(75)$ & $42(89)$ & $26(70)$ & $47(67)$ & \\
\hline 6 & $3(13)$ & $5(11)$ & $7(19)$ & $16(23)$ & \\
\hline 7 & $3(13)$ & $0(0)$ & $4(11)$ & $7(10)$ & \\
\hline Tumor size & & & & & 0.073 \\
\hline Range (median, cm) & $0.5-8(2.1)$ & $1-8(2.8)$ & $0.8-10(3.4)$ & $0.5-10(3.8)$ & \\
\hline $0.1-3.0 \mathrm{~cm}$ & $19(79)$ & $32(68)$ & $18(49)$ & $36(51)$ & \\
\hline $3.1-10.0 \mathrm{~cm}$ & $5(21)$ & $15(32)$ & $19(51)$ & $34(49)$ & \\
\hline
\end{tabular}

Abbreviations: TACE transcatheter arterial chemoembolization, Tx treatment, SABR stereotactic ablative radiotherapy, BCLC Barcelona Clinic Liver Cancer, CTP Child-Turcotte-Pugh

groups 1, 2, and 3, or between groups 3 and 4 . Nonetheless, group 1 patients had relatively lower BCLC stages and smaller tumors than the other groups, whereas those in group 2 had lower CTP scores and smaller tumors (Fig. 2).

\section{Overall survival}

The median follow up duration after the initial TACE for all patients was 35 months (range, 2-83 months). The OS rates at 2 and 5 years were $88 \%$ and $50 \%$ for group 1, $81 \%$ and $58 \%$ for group 2, $73 \%$ and $53 \%$ for group 3, and $54 \%$ and $28 \%$ for group 4 , respectively (Fig. 3). No significant differences in OS rates were observed among groups 1, 2, and 3. However, group 4 showed a significantly different OS rate compared with the other 3 groups (vs. group $1, P=0.010$; vs. group $2, P=0.001$; vs. group 3 , $P=0.040$; Table 2).

In univariate analysis, CTP score, tumor size, and treatment options were identified as significant prognostic factors for OS (Table 2). A CTP score of 7 was associated with significantly lower survival rates than CTP scores of 5 or 6 . Tumors $>3 \mathrm{~cm}$ had significantly lower survival rates than those $<3 \mathrm{~cm}$. In multivariate analysis, CTP score and treatment options were identified as significant prognostic factors. Group 4 showed significantly lower survival rates than
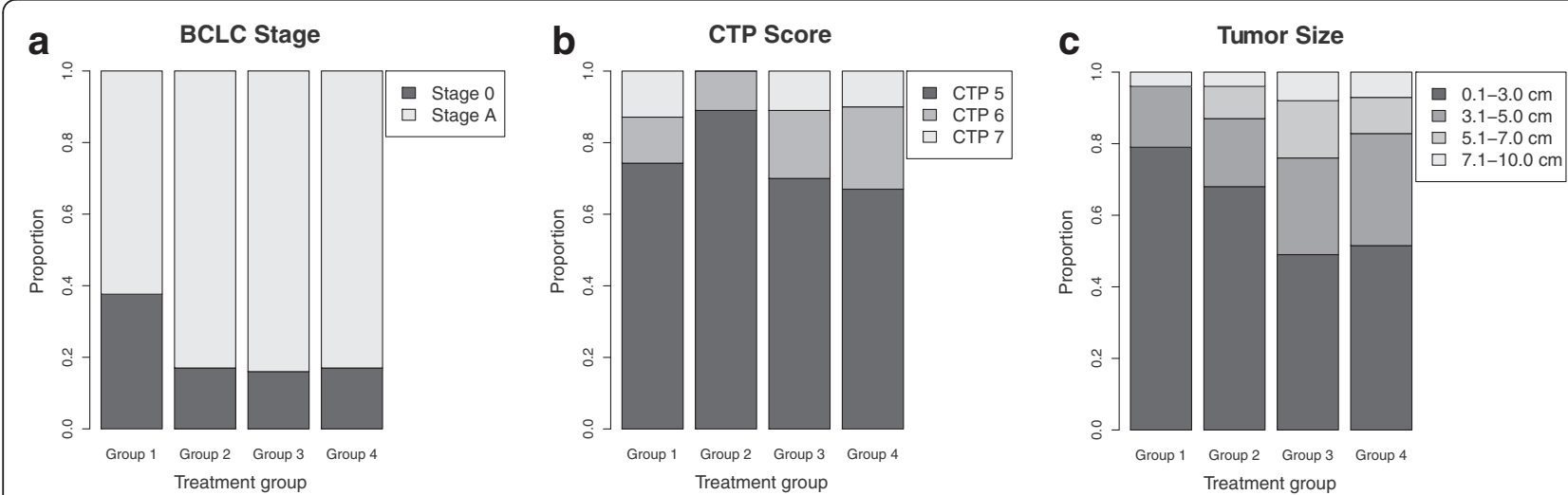

Fig. 2 Tumor characteristics of each treatment group. a By Barcelona Clinic Liver Cancer (BCLC) stage; (b) By Child-Turcotte-Pugh (CTP) score; (c) By tumor size 


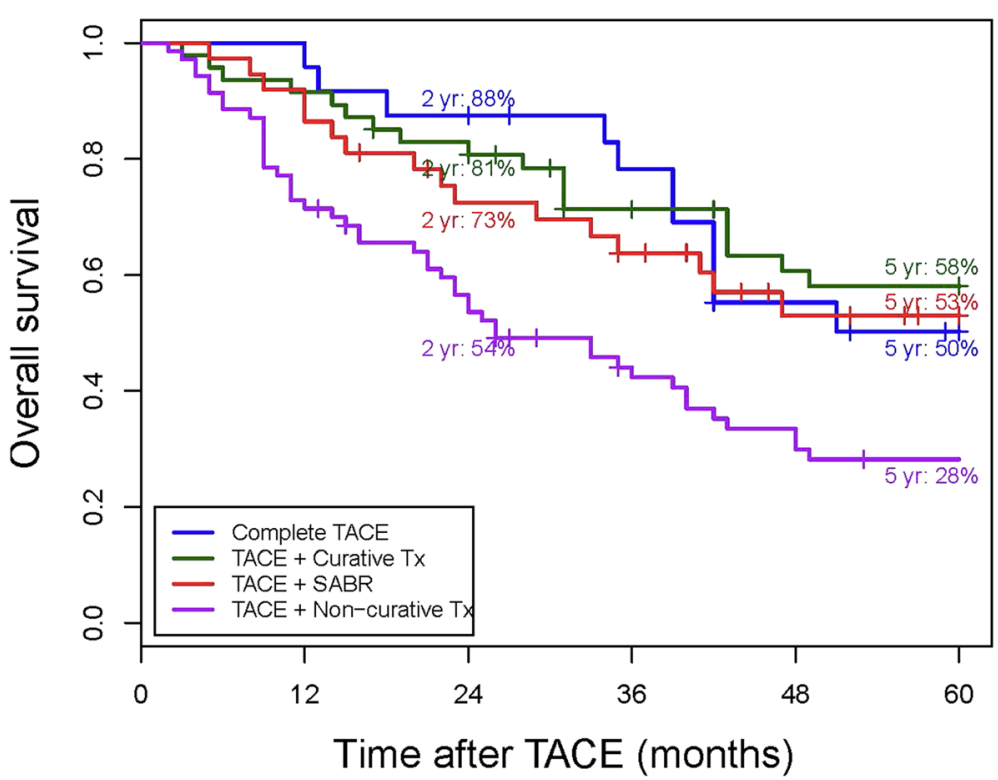

Fig. 3 Patients' overall survival for each treatment group from the time of the first TACE treatment. No significant differences in overall survival rates were observed among groups 1,2, and 3. However, group 4 showed a significantly inferior overall survival rate compared with the other 3 groups. Abbreviations: TACE, transcatheter arterial chemoembolization; Tx, treatment; SABR, stereotactic ablative radiotherapy

the other 3 groups, but no significant differences were observed among groups 1,2 , and 3 .

\section{Discussion}

In previous phase I and phase II studies conducted at our institute $[4,19]$, the feasibility of SABR for the treatment of primary $\mathrm{HCC}$ with incomplete response to TACE has been demonstrated with a 2-year LC rate of
$94.6 \%$ and a 2-year OS rate of $68.7 \%$. In the long term follow up study [20], LC and OS rates at 5 years were $82 \%$ and $39 \%$, respectively. Furthermore, at the last follow-up (4.5 years), patients receiving high-dose SABR ( $>54$ Gy) reported a $100 \% \mathrm{LC}$ rate and a $68 \%$ OS rate, which were comparable to RFA outcomes. Based on the results of these studies of SABR, HCC cases with a CTP score of $5-7$, total sum of tumor size $<10 \mathrm{~cm}$, and $\leq 3$

Table 2 Prognostic factors for overall survival

\begin{tabular}{|c|c|c|c|}
\hline \multirow[t]{2}{*}{ Factor } & \multirow{2}{*}{$\begin{array}{l}5 \text {-year } \\
\text { OS (\%) }\end{array}$} & \multirow{2}{*}{$\begin{array}{l}\text { Univariate analysis } \\
P \text {-value }\end{array}$} & \multirow{2}{*}{$\begin{array}{l}\text { Multivariate analysis } \\
\text { HR }(95 \% \mathrm{Cl})\end{array}$} \\
\hline & & & \\
\hline \multicolumn{4}{|l|}{ Treatment option } \\
\hline Complete TACE & 50 & & \\
\hline TACE + Curative Tx & 58 & 0.843 & $1.100(0.521-2.324)$ \\
\hline $\mathrm{TACE}+\mathrm{SABR}$ & 53 & 0.493 & $1.207(0.573-2.545)$ \\
\hline TACE + Non-curative Tx & 28 & 0.010 & $2.421(1.248-4.498)$ \\
\hline \multicolumn{4}{|l|}{$\mathrm{BCLC}$ stage } \\
\hline 0 & 54 & & \\
\hline A & 42 & 0.139 & - \\
\hline \multicolumn{4}{|l|}{ CTP score } \\
\hline 5 & 48 & & \\
\hline 6 & 33 & 0.128 & $1.037(0.622-1.729)$ \\
\hline 7 & 29 & 0.013 & $2.229(1.147-4.332)$ \\
\hline \multicolumn{4}{|l|}{ Tumor size (cm) } \\
\hline $0.1-3.0$ & 49 & & \\
\hline $3.1-10.0$ & 32 & 0.008 & - \\
\hline
\end{tabular}


nodules seem to be feasible for curative SABR. Therefore, to investigate whether the addition of SABR to TACE offers a survival benefit to HCC patients with incomplete response to TACE, we enrolled those meeting the above-mentioned eligibility criteria. In this study, the addition of SABR after incomplete TACE demonstrated significant survival benefits compared with additional non-curative treatments such as repeated TACE, sorafenib, and chemotherapy. Moreover, it also showed similar survival outcomes to those of the good prognostic groups, such as patients with complete response to TACE or those undergoing surgery with incomplete TACE.

TACE is widely adopted as the current standard of care for HCC patients at BCLC intermediate stage. Additionally, it might be indicated for patients with early stage HCCs that are unresectable or ineligible for local ablative therapies such as RFA or PEI due to tumor location or other medical conditions. Although complete response to TACE has not yet been defined in radiological findings, compact lipiodolisation is an important goal of TACE. Cabibbo et al. [25] reported that a complete radiological response after TACE significantly increases OS, and thus proposed utilizing the observation as a surrogate treatment endpoint. Kim et al. [26] indicated that compact lipiodol uptake following TACE predicted favorable survival outcomes for unresectable HCC. The 1year and 5-year OS rates of patients with compact lipiodolisation were $93 \%$ and $52 \%$, respectively, versus $61 \%$ and $17 \%$, respectively, in patients with noncompact lipiodolisation. Similarly, in our current study, the OS rates of group 1 patients with complete response to TACE at 1 and 5 years were $96 \%$ and $53 \%$, respectively. In addition, only $14 \%$ of the enrolled patients in this study achieved a complete response after 1-4 sessions of repeated TACE. Furthermore, approximately $40 \%$ of the patients were in stage 0 , and almost $80 \%$ had tumors $<3 \mathrm{~cm}$, suggesting that group 1 patients had relatively lower BCLC stages and smaller tumors than those in the incomplete response groups. Therefore, although group 3 patients, who received SABR after incomplete response to TACE, might have had poorer prognostic factors than group 1 patients, similar OS rates were observed between the 2 groups.

Since a compact radiological response after TACE significantly increases OS, technical improvements and refinements have been described for transarterial administrative methods [3, 27]. However, many patients still experience incomplete response after TACE, especially in cases of tumors $>3 \mathrm{~cm}$ [28]. Several studies [2932] have shown that the combination of TACE and other modalities, mainly surgery, RFA, PEI, or RT, was associated with higher survival rates. According to a prospective cohort study by Lee et al. [30], in which surgical resection after primary TACE was compared with TACE alone, the OS rate was significantly higher for the surgical resection group than for the TACE alone group (5year OS rate, $56 \%$ vs. $23 \%$ ). Studies on TACE plus RFA showed that patients in the TACE plus RFA group had better LC rates than patients in the TACE alone group [31], and better OS rates than patients in the RFA alone group [32]. TACE plus RFA have also been reported to provide similar OS rates to those achieved with surgical resection [33]. Several randomized trials were conducted to compare TACE plus PEI and TACE alone [34-36], suggesting that TACE plus PEI performed better than either TACE or PEI alone, and the reported 3-year OS rates for TACE plus PEI were approximately $35 \%-65 \%$. In our current study, group 2 patients underwent surgery, RFA, or PEI after incomplete TACE. This group only included patients with CTP class A. Our findings suggested that TACE might have been performed as a bridge therapy in patients who were initially eligible for curative treatments and subsequently underwent additional surgery. Also, group 2 patients had significantly lower CTP scores than the non-curative treatment group 4 patients. Therefore, group 2 patients might initially have had good prognostic factors, and our results also indicated a significant survival benefit in this group compared with group 4. Additionally, although not statistically significant, group 2 consisted of patients with relatively lower CTP scores compared to group 3, and smaller tumors compared to groups 3 and 4. Therefore, although group 3 patients might have had poorer prognostic factors than group 2 patients, similar OS rates were observed.

The benefit of additional 3D-CRT to incomplete TACE over TACE alone in unresectable HCC has also been reported. Meng et al. [10] conducted a meta-analysis of 17 trials, including 5 randomized controlled and 12 nonrandomized controlled studies. Although TACE plus RT showed significantly improved survival and tumor response compared with TACE alone in this meta-analysis, none of the 5 randomized trials reported the random allocation sequence in detail, and most of the original studies included were published in Chinese, making general acceptance challenging. On the other hand, in a retrospective study, Shim et al. [5] reported that a combination of 3D-CRT and incomplete TACE significantly improved survival rates compared with TACE alone. Their study included single tumors $\geq 5 \mathrm{~cm}$, and the 2year OS rates of the TACE plus RT and TACE alone groups were $37 \%$ and $14 \%$, respectively. The combination of SABR and TACE has previously been studied by Honda et al. [21]. Their results showed that SABR combined with TACE was safe and effective for loco-regional treatments, increasing both LC and OS compared with TACE alone. The 1-year and 3-year OS rates for the 
SABR group were $100 \%$ and $100 \%$, respectively, whereas the corresponding rates for the TACE group were $89 \%$, and $66 \%$, respectively. Although excellent OS rates were reported, patient selection was very strict, with only solitary tumors and small tumors $\leq 3 \mathrm{~cm}$ included. Our results also indicated a significant survival benefit of TACE plus SABR (group 3) over TACE alone (group 4) with similar baseline characteristics between the 2 groups.

There is not enough evidence to establish any combination therapy as a standard treatment for HCC after incomplete TACE. In our study, combining SABR to TACE offered similar survival outcomes to TACE and curative treatments, and survival benefits over repeated TACE, suggesting that SABR might be recommended as a treatment modality after TACE failure. Furthermore, SABR, being a noninvasive procedure, might be more advantageous than other invasive curative modalities such as surgery, RFA, or PEI. However, since this was a retrospective study, patients were not controlled with respect to variable prognostic factors. Additionally, the dose and fractionation schedules of SABR and the number of previous TACE sessions prior to SABR are still not well defined and are yet to be determined. Therefore, further randomized trials are needed to validate our results before SABR can be recommended routinely. Hence, based on the results of this study, a multicenter randomized controlled trial is being planned to investigate the potential benefits of SABR as an alternative modality in the treatment of HCC after incomplete TACE.

\section{Conclusions}

Patients treated with SABR after incomplete TACE had similar survival outcomes to those achieving complete response to TACE or those receiving curative treatments such as surgery, RFA, or PEI after incomplete TACE. The addition of SABR also had a significant survival benefit compared to repetitive TACE treatments. The present retrospective study showed that about $20 \%$ of the patients initially treated with TACE met the eligibility criteria for curative SABR treatments, and may benefit from further treatments of SABR. Therefore, SABR might be considered an alternative treatment modality following TACE failure.

\footnotetext{
Abbreviations

3D-CRT: three-dimensional conformal radiotherapy; AFP: alpha-fetoprotein; BCLC: Barcelona Clinic Liver Cancer; CT: computed tomography; CTP: Child-Turcotte-Pugh; GTV: gross tumor volume; HCC: hepatocellular carcinoma; ITV: internal target volume; LC: local control; LD: longest diameter; OS: overall survival; PEl: percutaneous ethanol injection; PTV: planning target volume; RFA: radiofrequency ablation; RT: radiotherapy; SABR: stereotactic ablative radiotherapy; TACE: transcatheter arterial chemoembolization.
}

\section{Competing interests}

The authors declare that they have no competing interests.

\section{Authors' contributions}

EKP participated in the design of the study, literature research, data acquisition, data analysis, data interpretation, statistical analysis, manuscript preparation, and manuscript editing. MSK contributed to study concepts, study design, definition of intellectual content, data analysis, data interpretation, manuscript editing, and manuscript review. All authors made substantial intellectual contributions to drafting the article, revising the article, data analysis, and data interpretation. All authors read and approved the final manuscript.

\section{Acknowledgements}

This work was supported by the National Nuclear R\&D Program of the Ministry of Science, ICT and Future Planning, Republic of Korea.

\section{Author details}

${ }^{1}$ Department of Radiation Oncology, Korea Cancer Center Hospital, Korea Institute of Radiological and Medical Sciences, 75 Nowon-ro, Nowon-gu, Seoul, Republic of Korea. ${ }^{2}$ Department of Internal Medicine, Korea Cancer Center Hospital, Korea Institute of Radiological and Medical Sciences, Seoul, Republic of Korea. ${ }^{3}$ Department of Surgery, Korea Cancer Center Hospital, Korea Institute of Radiological and Medical Sciences, Seoul, Republic of Korea. ${ }^{4}$ Department of Radiology, Korea Cancer Center Hospital, Korea Institute of Radiological and Medical Sciences, Seoul, Republic of Korea.

Received: 2 September 2015 Accepted: 1 February 2016

Published online: 19 February 2016

\section{References}

1. European Association For The Study Of The Liver, European Organisation For Research And Treatment Of Cancer. EASL-EORTC clinical practice guidelines: management of hepatocellular carcinoma. J Hepatol. 2012;56: 908-43.

2. Llovet JM, Bruix J. Systematic review of randomized trials for unresectable hepatocellular carcinoma: Chemoembolization improves survival. Hepatology. 2003;37:429-42.

3. Lencioni R. Chemoembolization for hepatocellular carcinoma. Semin Oncol. 2012;39:503-9.

4. Kang JK, Kim MS, Cho CK, Yang KM, Yoo HJ, Kim JH, et al. Stereotactic body radiation therapy for inoperable hepatocellular carcinoma as a local salvage treatment after incomplete transarterial chemoembolization. Cancer. 2012; 118:5424-31.

5. Shim SJ, Seong J, Han KH, Chon CY, Suh CO, Lee JT. Local radiotherapy as a complement to incomplete transcatheter arterial chemoembolization in locally advanced hepatocellular carcinoma. Liver Int. 2005;25:1189-96.

6. Ingold JA, Reed GB, Kaplan HS, Bagshaw MA. Radiation hepatitis. Am J Roentgenol Radium Ther Nucl Med. 1965;93:200-8.

7. Pan CC, Kavanagh BD, Dawson LA, Li XA, Das SK, Miften M, et al. Radiationassociated liver injury. Int J Radiat Oncol Biol Phys. 2010;76:S94-100.

8. Dawson LA, McGinn CJ, Normolle D, Ten Haken RK, Walker S, Ensminger W, et al. Escalated focal liver radiation and concurrent hepatic artery fluorodeoxyuridine for unresectable intrahepatic malignancies. J Clin Oncol. 2000;18:2210-8.

9. Seong J, Park HC, Han KH, Chon CY. Clinical results and prognostic factors in radiotherapy for unresectable hepatocellular carcinoma: a retrospective study of 158 patients. Int J Radiat Oncol Biol Phys. 2003;55:329-36.

10. Meng MB, Cui YL, Lu Y, She B, Chen Y, Guan YS, et al. Transcatheter arterial chemoembolization in combination with radiotherapy for unresectable hepatocellular carcinoma: a systematic review and meta-analysis. Radiother Oncol. 2009;92:184-94.

11. Potters L, Kavanagh B, Galvin JM, Hevezi JM, Janjan NA, Larson DA, et al. American Society for Therapeutic Radiology and Oncology (ASTRO) and American College of Radiology (ACR) practice guideline for the performance of stereotactic body radiation therapy. Int J Radiat Oncol Biol Phys. 2010;76:326-32.

12. Kim MS, Kim W, Park $H$, Kim HJ, Lee E, Jung JH, et al. Radiobiological mechanisms of stereotactic body radiation therapy and stereotactic radiation surgery. Radiat Oncol J. 2015;33:265-275.

13. Andolino DL, Johnson CS, Maluccio M, Kwo P, Tector AJ, Zook J, et al. Stereotactic body radiotherapy for primary hepatocellular carcinoma. Int J Radiat Oncol Biol Phys. 2011;81:e447-53. 
14. Louis C, Dewas S, Mirabel X, Lacornerie T, Adenis A, Bonodeau F, et al. Stereotactic radiotherapy of hepatocellular carcinoma: preliminary results. Technol Cancer Res Treat. 2010;9:479-87.

15. Tse RV, Hawkins M, Lockwood G, Kim JJ, Cummings B, Knox J, et al. Phase I study of individualized stereotactic body radiotherapy for hepatocellular carcinoma and intrahepatic cholangiocarcinoma. J Clin Oncol. 2008;26:657-64.

16. Sanuki N, Takeda A, Oku Y, Mizuno T, Aoki Y, Eriguchi T, et al. Stereotactic body radiotherapy for small hepatocellular carcinoma: a retrospective outcome analysis in 185 patients. Acta Oncol. 2014;53:399-404.

17. Sanuki N, Takeda A, Kunieda E. Role of stereotactic body radiation therapy for hepatocellular carcinoma. World J Gastroenterol. 2014;20:3100-11.

18. Yamashita H, Onishi J, Matsumoto Y, Murakami N, Matsuo Y, Nomiya T, et al. Local effect of stereotactic body radiotherapy for primary and metastatic liver tumors in 130 Japanese patients. Radiat Oncol. 2014;9:112.

19. Seo YS, Kim MS, Yoo SY, Cho CK, Choi CW, Kim JH, et al. Preliminary result of stereotactic body radiotherapy as a local salvage treatment for inoperable hepatocellular carcinoma. J Surg Oncol. 2010;102:209-14.

20. Jang WI, Kim MS, Bae SH, Cho CK, Yoo HJ, Seo YS, et al. High-dose stereotactic body radiotherapy correlates increased local control and overall survival in patients with inoperable hepatocellular carcinoma. Radiat Oncol. 2013;8:250.

21. Honda Y, Kimura T, Aikata H, Kobayashi T, Fukuhara T, Masaki K, et al. Stereotactic body radiation therapy combined with transcatheter arterial chemoembolization for small hepatocellular carcinoma. J Gastroenterol Hepatol. 2013;28:530-6.

22. Bruix J, Sherman M, American Association for the Study of Liver Diseases. Management of hepatocellular carcinoma: an update. Hepatology. 2011:53:1020-2

23. Kudo M, Izumi N, Kokudo N, Matsui O, Sakamoto M, Nakashima O, et al. Management of hepatocellular carcinoma in Japan: Consensus-Based Clinical Practice Guidelines proposed by the Japan Society of Hepatology (JSH) 2010 updated version. Dig Dis. 2011;29:339-64.

24. Llovet JM, Real MI, Montana X, Planas R, Coll S, Aponte J, et al. Arterial embolisation or chemoembolisation versus symptomatic treatment in patients with unresectable hepatocellular carcinoma: a randomised controlled trial. Lancet. 2002:359:1734-9.

25. Cabibbo G, Genco C, Di Marco V, Barbara M, Enea M, Parisi P, et al. Predicting survival in patients with hepatocellular carcinoma treated by transarterial chemoembolisation. Aliment Pharmacol Ther. 2011;34:196-204.

26. Kim DY, Ryu HJ, Choi JY, Park JY, Lee DY, Kim BK, et al. Radiological response predicts survival following transarterial chemoembolisation in patients with unresectable hepatocellular carcinoma. Aliment Pharmacol Ther. 2012;35:1343-50

27. Burrel M, Reig M, Forner A, Barrufet M, de Lope CR, Tremosini S, et al. Survival of patients with hepatocellular carcinoma treated by transarterial chemoembolisation (TACE) using Drug Eluting Beads. Implications for clinical practice and trial design. J Hepatol. 2012;56:1330-5.

28. Higuchi T, Kikuchi M, Okazaki M. Hepatocellular carcinoma after transcatheter hepatic arterial embolization. A histopathologic study of 84 resected cases. Cancer. 1994;73:2259-67.

29. Liao M, Huang J, Zhang T, Wu H. Transarterial chemoembolization in combination with local therapies for hepatocellular carcinoma: a metaanalysis. PLoS One. 2013;8:e68453.

30. Lee HS, Kim KM, Yoon JH, Lee TR, Suh KS, Lee KU, et al. Therapeutic efficacy of transcatheter arterial chemoembolization as compared with hepatic resection in hepatocellular carcinoma patients with compensated liver function in a hepatitis B virus-endemic area: a prospective cohort study. J Clin Oncol. 2002;20:4459-65.

31. Yang $P$, Liang $M$, Zhang $Y$, Shen B. Clinical application of a combination therapy of lentinan, multi-electrode RFA and TACE in HCC. Adv Ther. 2008; 25:787-94.

32. Peng ZW, Zhang YJ, Chen MS, Xu L, Liang HH, Lin XJ, et al. Radiofrequency ablation with or without transcatheter arterial chemoembolization in the treatment of hepatocellular carcinoma: a prospective randomized trial. J Clin Oncol. 2013;31:426-32

33. Kagawa T, Koizumi J, Kojima S, Nagata N, Numata M, Watanabe N, et al. Transcatheter arterial chemoembolization plus radiofrequency ablation therapy for early stage hepatocellular carcinoma: comparison with surgical resection. Cancer. 2010;116:3638-44.

34. Kato T, Saito Y, Niwa M, Ishiguro J, Ogoshi K. Combination therapy of transcatheter chemoembolization and percutaneous ethanol injection therapy for unresectable hepatocellular carcinoma. Cancer Chemother Pharmacol. 1994;33(Suppl):S115-8.

35. Bartolozzi C, Lencioni R, Caramella D, Vignali C, Cioni R, Mazzeo S, et al. Treatment of large HCC: transcatheter arterial chemoembolization combined with percutaneous ethanol injection versus repeated transcatheter arterial chemoembolization. Radiology. 1995;197:812-8.

36. Becker G, Soezgen T, Olschewski M, Laubenberger J, Blum HE, Allgaier HP. Combined TACE and PEI for palliative treatment of unresectable hepatocellular carcinoma. World J Gastroenterol. 2005:11:6104-9.

\section{Submit your next manuscript to BioMed Central and we will help you at every step:}

- We accept pre-submission inquiries

- Our selector tool helps you to find the most relevant journal

- We provide round the clock customer support

- Convenient online submission

- Thorough peer review

- Inclusion in PubMed and all major indexing services

- Maximum visibility for your research

Submit your manuscript at www.biomedcentral.com/submit
) Biomed Central 